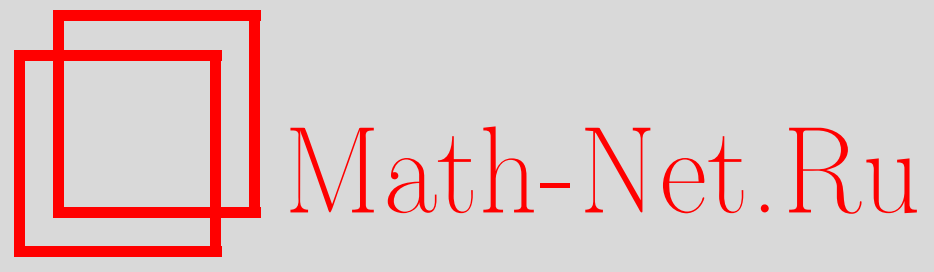

В. А. Воблый, О перечислении помеченных связных бициклических и трициклических графов без мостов, $M a$ тем. заметки, 2012, том 91, выпуск 2, 308-311

DOI: https://doi.org/10.4213/mzm8969

Использование Общероссийского математического портала Math-Net.Ru подразумевает, что вы прочитали и согласны с пользовательским соглашением http://www . mathnet.ru/rus/agreement

Параметры загрузки:

IP : 54.162 .85 .209

26 апреля 2023 г., 05:12:25






\section{О перечислении помеченных связных бициклических и трициклических графов без мостов}

\section{В. А. Воблый}

Рассматриваются неориентированные простые связные графы. Мостом связного графа называется его ребро, после удаления которого граф становится несвязным. Гладким графом называется граф без висячих вершин. Очевидно, множество графов без мостов является подмножеством множества гладких графов.

Гладкие графы перечислил Райт [1]. Хенлон и Робинсон [2] перечислили как непомеченные, так и помеченные графы без мостов. Для производящей функции помеченных графов они получили функциональное уравнение, а также нелинейное уравнение с частными производными. Однако их формулы громоздки и не приведены к виду, удобному для вычисления. Вероятно, поэтому в знаменитой "Онлайн-энциклопедии целочисленных последовательностей” Слоэна [3] есть только данные о числе непомеченных графов без мостов.

Целью данной работы было получение явных формул для числа помеченных связных бициклических и трициклических графов без мостов, а также соответствующей асимптотики для числа таких графов с большим числом вершин.

Напомним, что точкой сочленения связного графа называется его вершина, после удаления которой вместе с инцидентными ей ребрами граф становится несвязным. Блоком называется связный граф без точек сочленения, а также максимальный связный нетривиальный подграф, не имеющий точек сочленения [4; с. 200]. Под унициклическим, биииклическим и трициклическим графом понимается связный граф с цикломатическим числом равным соответственно 1,2 и 3 . Пусть при $k \geqslant 0 u(n, n+k), l(n, n+k), v(n, n+k)$ - число помеченных блоков с $n$ вершинами и $n+k$ ребрами, число помеченных связных графов без мостов с $n$ вершинами и $n+k$ ребрами и число помеченных гладких графов с $n$ вершинами и $n+k$ ребрами соответственно. Все такие графы имеют цикломатическое число, равное $k+1$. Обозначим через $U_{k}(w), L_{k}(w)$ экспоненциальные производящие функции:

$$
U_{k}(w)=\sum_{n=3}^{\infty} u(n, n+k) \frac{w^{n}}{n !}, \quad L_{k}(w)=\sum_{n=3}^{\infty} l(n, n+k) \frac{w^{n}}{n !} .
$$

Теорема 1. Число помеченных связных бициклических графов без мостов с $n$ вериинами при $n \geqslant 4$ равно

$$
l(n, n+1)=\frac{n !}{24}\left(n^{2}+2 n-18\right) .
$$

ДокАЗАТЕльСтво. Так как блок содержит как минимум один цикл, то бициклический граф без мостов является либо бициклическим блоком, либо графом с одной точкой сочленения, у которого каждый из двух блоков - простой цикл. Пусть $\bar{l}(n, n+1)$ - число бициклических графов без мостов, не являющихся блоками. Тогда

$$
l(n, n+1)=u(n, n+1)+\bar{l}(n, n+1) .
$$

Райт [1] нашел, что при $n \geqslant 4$

$$
u(n, n+1)=\frac{n !(n-3)(n+2)}{24} .
$$

Пусть

$$
\bar{L}_{1}(w)=\sum_{n=3}^{\infty} \bar{l}(n, n+1) \frac{w^{n}}{n !}
$$


Известно [4; с. 201-202], что производящая функция корневых графов с непомеченным корнем получается дифференцированием производящей функции таких же некорневых графов со всеми помеченными вершинами. Поэтому имеем $\bar{L}_{1}(w)=(1 / 2) w\left(U_{0}^{\prime}(w)\right)^{2}$, где множитель $w$ появляется для того, чтобы пометить точку сочленения после склейки корней двух блоков, а множитель $1 / 2$ - из-за возможности перестановки двух блоков. Поскольку производящая функция помеченных унициклических блоков совпадает с производящей функцией простых циклов, то

$$
U_{0}(w)=\sum_{n=3}^{\infty} \frac{1}{2}(n-1) ! \frac{w^{n}}{n !}=-\frac{1}{2} \ln (1-w)-\frac{w}{2}-\frac{w^{2}}{4} \quad \text { и } \quad U_{0}^{\prime}(w)=\frac{w^{2}}{2(1-w)} .
$$

С помощью известного разложения (см., например, [5; с. 709])

$$
(1-w)^{-m-1}=\sum_{n=0}^{\infty}\left(\begin{array}{c}
m+n \\
m
\end{array}\right) w^{n}
$$

получим

$$
\bar{L}_{1}(w)=\frac{w^{5}}{8(1-w)^{2}}=\frac{w^{5}}{8} \sum_{n=0}^{\infty}\left(\begin{array}{c}
n+1 \\
1
\end{array}\right) w^{n}=\frac{1}{8} \sum_{n=5}^{\infty}(n-4) w^{n}
$$

и, следовательно, окончательно найдем

$$
l(n, n+1)=n !\left(\frac{1}{24}(n-3)(n+2)+\frac{n-4}{8}\right)=\frac{n !}{24}\left(n^{2}+2 n-18\right) .
$$

Из теоремы 1 непосредственно вытекает

СлЕДСтвиЕ 1. При $n \rightarrow \infty$ верно асимптотическое равенство

$$
l(n, n+1) \sim \frac{n^{2}}{24} n ! .
$$

Зададим на множестве помеченных гладких бициклических графов равномерное распределение. Пусть $P_{1}(n)$ - вероятность того, что помеченный гладкий бициклический граф с $n$ вершинами не имеет мостов.

СлЕДСТвиЕ 2. При $n \rightarrow \infty$ верно асимптотическое равенство

$$
P_{1}(n) \sim \frac{2}{5}
$$

ДокАзАтельство. Так как $P_{1}(n)=l(n, n+1) / v(n, n+1)$, с учетом формулы

$$
v(n, n+1)=\frac{n !(n-3)(5 n-8)}{48},
$$

найденной в работе Райта [1], получим доказываемое асимптотическое равенство.

Теорема 2. Число помеченных свлзных триииклических графов без мостов с $n$ вершинами при $n \geqslant 5$ равно

$$
l(n, n+2)=\frac{n !}{1152}\left(n^{5}+7 n^{4}-59 n^{3}-91 n^{2}+550 n+360\right) .
$$

ДоказАтельство. Разобьем множество помеченных трициклических графов без мостов на 4 непересекающихся подмножества. Тогда для соответствующих производящих функций имеем равенство

$$
L_{2}(w)=Q_{1}(w)+Q_{2}(w)+Q_{3}(w)+Q_{4}(w) .
$$


Первое подмножество состоит из трициклических блоков. Поэтому $Q_{1}(w)=U_{2}(w)$. Райт [1] вывел формулу

$$
u(n, n+2)=\frac{n !}{1152}(n-3)\left(n^{4}+4 n^{3}-15 n^{2}-46 n-40\right) .
$$

Второе подмножество состоит из графов с одной точкой сочленения, у которых каждый блок является простым циклом. Такие графы получаются путем склеивания непомеченных корней трех помеченных простых циклов. Поэтому $Q_{2}(w)=(1 / 6) w\left(U_{0}^{\prime}(w)\right)^{3}$. Здесь множитель $w$ присутствует для пометки точки сочленения после склейки корней трех блоков, а множитель $1 / 6$ - из-за возможности перестановки блоков.

Третье подмножество состоит из графов с одной точкой сочленения, у которых один блок бициклический, а второй - простой цикл. Следовательно, имеем $Q_{3}(w)=w U_{1}^{\prime}(w) \times$ $U_{0}^{\prime}(w)$. Эти графы получаются путем склеивания непомеченных корней двух помеченных блоков, а множитель $w$ добавляется для пометки точки сочленения.

Четвертое подмножество состоит из графов с двумя точками сочленения, у которых каждый блок является простым циклом. Поэтому $Q_{4}(w)=(1 / 2) w^{2} U_{0}^{\prime \prime}(w)\left(U_{0}^{\prime}(w)\right)^{2}$. Здесь присутствует множитель $1 / 2$ - из-за возможности перестановки крайних блоков, множитель $w^{2}$ вводится для пометки двух точек сочленения после склейки корней блоков, а $U_{0}^{\prime \prime}(w)$ - производящая функция простого цикла с двумя непомеченными корнями.

Райт [1] нашел, что

$$
U_{1}(w)=\frac{1}{12}(1-\theta)^{4}\left(\theta^{-3}+2 \theta^{-2}\right),
$$

где $\theta=1-w$. Следовательно, получим

$$
U_{1}(w)=\frac{w^{4}(3-2 w)}{12(1-w)^{3}}, \quad U_{1}^{\prime}(w)=\frac{4 w^{5}-13 w^{4}+12 w^{3}}{12(1-w)^{4}}, \quad U_{0}^{\prime \prime}(w)=\frac{2 w-w^{2}}{2(1-w)^{2}} .
$$

Обозначим через $\bar{l}(n, n+2)$ - число трициклических графов без мостов, не являющихся блоками, и пусть

$$
\bar{L}_{2}(w)=\sum_{n=3}^{\infty} \bar{l}(n, n+2) \frac{w^{n}}{n !}
$$

- соответствующая производящая функция. Тогда имеем

$$
\begin{aligned}
\bar{L}_{2}(w) & =Q_{2}(w)+Q_{3}(w)+Q_{4}(w) \\
& =\frac{w^{7}}{48(1-w)^{3}}+\frac{w^{6}\left(4 w^{2}-13 w+12\right)}{24(1-w)^{5}}+\frac{w^{7}(2-w)}{16(1-w)^{4}} \\
& =\frac{w^{7}}{12(1-w)^{3}}+\frac{w^{7}}{16(1-w)^{4}}+\frac{w^{6}}{6(1-w)^{3}}+\frac{5 w^{6}}{24(1-w)^{4}}+\frac{w^{6}}{8(1-w)^{5}} .
\end{aligned}
$$

C помощью разложения (1) найдем

$$
\begin{aligned}
\bar{L}_{2}(w)=\sum_{n=0}^{\infty} & \left(\frac{1}{12}\left(\begin{array}{c}
n+2 \\
2
\end{array}\right)+\frac{1}{16}\left(\begin{array}{c}
n+3 \\
3
\end{array}\right)\right) w^{n+7}+\sum_{n=0}^{\infty} \frac{1}{6}\left(\begin{array}{c}
n+2 \\
2
\end{array}\right) w^{n+6} \\
& +\sum_{n=0}^{\infty}\left(\frac{5}{24}\left(\begin{array}{c}
n+3 \\
3
\end{array}\right)+\frac{1}{8}\left(\begin{array}{c}
n+4 \\
4
\end{array}\right)\right) w^{n+6}=\sum_{n=7}^{\infty} \frac{(n-5)(n-6)}{24} w^{n} \\
& +\sum_{n=7}^{\infty} \frac{(n-4)(n-5)(n-6)}{96} w^{n}+\sum_{n=6}^{\infty} \frac{(n-4)(n-5)}{12} w^{n} \\
& +\sum_{n=6}^{\infty}\left(\frac{5(n-3)(n-4)(n-5)}{144}+\frac{(n-2)(n-3)(n-4)(n-5)}{192}\right) w^{n}
\end{aligned}
$$




$$
=\sum_{n=6}^{\infty} \frac{\left(3 n^{4}-16 n^{3}-45 n^{2}+226 n+120\right)}{576} w^{n} .
$$

Учитывая, что $l(n, n+2)=u(n, n+2)+\bar{l}(n, n+2)$, получим утверждение теоремы.

Из теоремы 2 очевидным образом вытекает

СледСтвиЕ 3. При $n \rightarrow \infty$ верно асимптотическое равенство

$$
l(n, n+2) \sim \frac{n^{5}}{1152} n !
$$

Зададим на множестве помеченных гладких трициклических графов равномерное распределение. Пусть $P_{2}(n)$ - вероятность того, что помеченный гладкий трициклический граф с $n$ вершинами не имеет мостов.

СлЕДСТвиЕ 4. При $n \rightarrow \infty$ верно асимптотическое равенство

$$
P_{2}(n) \sim \frac{1}{3}
$$

ДоказАтельство. Райт [6] при $n \rightarrow \infty$ дает асимптотическую формулу $v(n, n+2) \sim$ $\left(n^{5} / 384\right) n$ !. Так как $P_{2}(n)=l(n, n+2) / v(n, n+2)$, имеем доказываемую асимптотику.

В заключение отметим, что Дмитриев [7] другим способом перечислял помеченные 2-реберно-связные графы (графы без мостов), но не опубликовал свои результаты.

Автор благодарит рецензента за замечания и предложения по улучшению изложения результатов работы.

\section{СПИСОК ЦИТИРОВАННОЙ ЛИТЕРАТУРЫ}

[1] E. M. Wright, J. Graph Theory, 2:4 (1978), 299-305. [2] P. Hanlon, R. W. Robinson, J. Combin. Theory Ser. B, 33:3 (1982), 276-305. [3] The on-line encyclopedia of integer sequences, http://www.research.att.com/ ${ }^{\sim}$ njas/sequences. [4] В. Н. Сачков, Введение в комбинаторные методы дискретной математики, МЦНМО, М., 2004. [5] А. П. Прудников, Ю.А. Брычков, О.И. Маричев, Интегралы и ряды. Элементарные функиии, Наука, M., 1981. [6] E. M. Wright, Proc. Roy. Soc. Edinburgh Sect. A, 91:3-4 (1982), 205-212. [7] Е.Ф. Дмитриев, Перечисление отмеченных графов с данными структурными свойствами, Автореферат дис. ... канд. физ.-матем. наук, Ин-т матем. АН БССР, Минск, 1986.

\section{В. А. Воблый}

Поступило

E-mail: vitvobl@yandex.ru

12.07 .2010

Исправленный вариант

13.05 .2011 